\title{
La diplomatie en images. Discours politique et mythe technocratique dans la bande dessinée Quai d'Orsay (tome I)
}

Sébastien Ségas

\section{(2) OpenEdition \\ Journals}

Édition électronique

URL : https://journals.openedition.org/mots/20692

DOI : $10.4000 /$ mots.20692

ISSN : 1960-6001

Éditeur

ENS Éditions

\section{Édition imprimée}

Date de publication : 15 septembre 2012

Pagination : $61-78$

ISBN : 9782847883732

ISSN : 0243-6450

Référence électronique

Sébastien Ségas, «La diplomatie en images. Discours politique et mythe technocratique dans la bande dessinée Quai d'Orsay (tome I) ", Mots. Les langages du politique [En ligne], 99 | 2012, mis en ligne le 15 septembre 2014, consulté le 22 avril 2022. URL : http://journals.openedition.org/mots/20692 DOI : https://doi.org/10.4000/mots.20692 


\section{La diplomatie en images. Discours politique et mythe technocratique dans la bande dessinée Quai d'Orsay (tome l)}

Gros succès de librairie, la bande dessinée Quai d'Orsay. Chroniques diplomatiques, tome I1 (Blain, Lanzac, 2010) a beaucoup fait parler d'elle. Une partie de ce succès, au-delà des qualités artistiques indéniables de l'œuvre, découle de son récit «à clef». En effet, ce livre décrit une histoire directement inspirée de la réalité, tout en la camouflant derrière des pseudonymes et en déformant certains événements. La référence à la réalité y est à la fois travestie et évidente, l'œuvre ayant pour objectif de montrer ce qu'elle prétend cacher. Une clef de lecture permet au lecteur non averti de comprendre de quoi et surtout de qui il est question. Cette clef est ici fournie, singularité permise par le dispositif de la BD, grâce au dessin : le ministre de Quai d'Orsay (appelé Alexandre Taillard de Vorms) ressemble trait pour trait à Dominique de Villepin. Le texte renforce cette impression : les péripéties diplomatiques qui émaillent le texte, impliquant, comme chez Hergé, des pays fictifs, ne sont pas sans rappeler les problèmes que le véritable Dominique de Villepin a dû traiter dans l'exercice de ses fonctions au Quai d'Orsay de 2002 à 2004. De fait, cette BD aborde la politique non pas sous l'angle exclusif de la caricature ${ }^{2}$ (même si celle-ci est loin d'être absente) ou sous l'angle de l'histoire-fiction, mais plutôt sous celui du témoignage. Quai d'Orsay se distingue ainsi fortement d'autres œuvres de BD contemporaines comme La face karchée de Sarkozy (Cohen, Malka, 2007), pour le versant satirique, ou J'ai pas tué de Gaulle mais ça a bien failli (Heitz, 2010), pour celui de l'histoire mise en fiction. Le témoignage qui fonde l'œuvre est celui d'un insider : le scénariste (Lanzac), qui signe sous un pseudonyme, a en effet appartenu au cabinet de Dominique de Villepin lors du passage de celui-ci au ministère des Affaires étrangères.

L’histoire narrée dans Quai d'Orsay est celle d'une éducation (à la) politique.

1. L'analyse développée ici ne porte pas sur le second tome de Quai d'Orsay paru en décembre 2011, alors que s'achevait la rédaction de cet article.

2. Sur ce point, voir la contribution synthétique de Marc Bonhomme, 2010. 
Dès les premières pages, le lecteur suit le parcours d'un jeune homme (Arthur Vlaminck), novice en politique, qui va rapidement être engagé au cabinet d'un ministre des Affaires étrangères (Alexandre Taillard de Vorms) pour être chargé d'une mission que le ministre définit comme capitale : «le langage»3 (p.7). Cette mission consiste en fait à rédiger des discours publics et des fiches pour le ministre. Le jeune homme occupe donc la fonction d' «écrivant» (OllivierYanniv, 2003) de la parole ministérielle, position qui l'amène à travailler à la fois avec le ministre et avec les conseillers thématiques du cabinet « qui aident le ministre à "penser" politiquement le problème que son ministère prend en charge» (Nollet, 2006, p. 165).

Arthur Vlaminck, au début du récit, apparaît à la fois naîf et idéaliste. Plutôt de gauche, semble-t-il4, il est séduit par les ambitions et la "vision » géostratégique du ministre, pourtant de droite. Au fil du récit, le jeune novice découvre que le cabinet n'est pas une équipe soudée défendant les intérêts de la France et une vision de l'équilibre géopolitique qui lui serait propre, mais une arène où s'affrontent des intérêts individuels divergents. Ainsi, le héros découvre qu'une de ses collègues, après lui avoir déclaré tout le bien qu'elle pensait d'un de ses discours, est, "dans son dos», venue critiquer son travail devant le ministre. Vlaminck découvre également que le ministre qu'il tient pour un visionnaire est aussi un obsédé du discours qui semble largement se désintéresser des aspects concrets de sa mission. D'ailleurs, les projets de discours du novice en politique sont régulièrement retoqués par Taillard de Vorms, non pour des raisons de fond mais de forme (défaut de structuration, d'énergie, de souffle, etc.). Le novice comprend progressivement que son travail consiste moins à se confronter aux problèmes diplomatiques concrets qu'à construire un discours à même de concilier les exigences souvent contradictoires des experts du cabinet (conseiller MoyenOrient, conseiller Europe, conseiller Amérique), de l'entourage intellectuel du ministre (philosophes, poètes) et, last but not least, du ministre lui-même.

Au fur et à mesure du récit, le rapport du héros au ministre se complexifie, passant de la fascination à un mélange d'attirance et de répulsion (le ministre apparaît même sous les traits de Dark Vadors dans un fantasme de Vlaminck

3. La notion de "langage» constitue une catégorie «indigène», au sens où elle fait partie des concepts que les membres de cabinet utilisent pour décrire leurs activités. Dans le lexique des activités diplomatiques, cette catégorie prend un sens particulier : «le terme désigne un instrument de la production collective de l'information par les diplomates et leurs collaborateurs, en vue de participer à la construction de l'image publique du cadre d'action de la politique étrangère, sans toutefois qu'il y ait nécessairement de relations avec les journalistes» (Ollivier-Yaniv, 2011, p. 61).

4. Le récit donne un indice indirect au lecteur de cet ancrage d'Arthur Vlaminck à gauche. Quand le héros annonce à sa compagne qu'il va travailler pour Taillard de Vorms (p. 9), celle-ci rétorque : «Mais c'est un mec de droite». Ce à quoi Vlaminck répond : «C'est un type ouvert, il a choisi un directeur de cabinet de gauche». Pour reprendre les catégories définies par Ollivier-Yaniv (2003), Arthur Vlaminck ne semble pas correspondre au profil de l'écrivant «militant», dont la légitimité est avant tout politique, mais plutôt à celui de l' "agrégé », dont la légitimité se fonde avant tout sur la maîtrise de compétences universitaires ou intellectuelles.

5. Dans la saga Star Wars de George Lucas, Dark Vador est à la fois le général en chef des armées 
qui occupe la dernière planche). En revanche, le héros trouve dans le discret directeur de cabinet Claude Maupas, homme de dossiers et d'action, un soutien constant. Si Quai d'Orsay est l'histoire d'un désenchantement, voire d'un déniaisement politique (celui de Vlaminck), cette histoire est structurée par l'opposition entre le charisme, le charme un peu creux d'un homme (Taillard de Vorms) et le sérieux un brin austère d'un autre (Maupas).

Notre analyse portera moins sur ce récit en lui-même que sur la façon dont il est narré, non pas sur la fiction mais sur le travail de fictionnalisation. L'idée que nous défendrons ici est que la façon dont le récit est agencé participe de la construction de la signification (ainsi une même histoire racontée par des conteurs différents n'est pas reçue de la même façon). Pour paraphraser Marshall Mac Luhan (1977), le message est en partie dans le médium : le choix des dialogues, la façon de dessiner, d'agencer les planches participent du travail de fictionnalisation et véhiculent un message à la fois sur les personnages centraux et sur l'activité diplomatique. Plus précisément, nous tenterons de montrer comment, à travers la mise en image d'une opposition entre le ministre des Affaires étrangères et son directeur de cabinet, s'opèrent à la fois une critique de la communication politique et une valorisation d'une approche technique, bureaucratique, discrète, de l'action diplomatique.

\section{À la recherche du bon niveau d'analyse pour une bande dessinée : phylactères, graphiations et «tressages »}

S'attaquer à l'analyse du travail de fictionnalisation dans une bande dessinée implique de se confronter à la particularité de ce médium. En effet, la bande dessinée ne peut se prêter à une simple analyse textuelle qui serait, dans ce cas, incroyablement réductrice. La signification n'est pas entièrement contenue dans le texte mais naît de la rencontre entre les images et les dialogues saisis dans les phylactères : «Il n'est question ni de légendes ni d'illustrations, mais bien d'une véritable complémentarité entre le lisible et le visible, deux instances qui assument chacune leur part de narrativité »(Peeters, 1993, p. 26). À l'instar du cinéma, la bande dessinée est une forme d'expression artistique qui combine discours et image et qui, de facto, interroge les limites du concept de narration créé à l'origine pour étudier des formes littéraires de récit. Dans le champ des études cinématographiques, André Gaudreault et François Jost (1990) ont proposé d'adapter ce concept aux formes non littéraires en distinguant, dans l'activité d'énonciation narrative, la narration proprement dite (un discours que l'on peut imputer, plus ou moins facilement, à un narrateur qui donne son point

de l'Empire (les forces du Mal) et le père du héros, lequel se situe, bien entendu, du bon côté de la «force ». Il constitue à ce titre une figure profondément ambiguë qui tente d'entraîner son fils vers «le côté obscur» avant de finalement l'aider à tuer l’Empereur. 
de vue sur les événements du récit et les personnages) et la monstration (où le point de vue du narrateur laisse la place aux dialogues et à l'action des personnages qui prennent en charge la narration). Pour ces auteurs, il s'agit moins d'opposer des formes de narration incompatibles entre elles (comme on pourrait opposer la littérature au théâtre) que d'étudier les œuvres cinématographiques en tant qu'instances simultanément narratives et monstratives. S'inspirant de ces travaux pour les importer dans le champ de la bande dessinée, Philippe Marion (1993) a créé le concept de graphiation : au travail du metteur en scène qui cherche à «faire voir», à «montrer» un récit, correspond celui de l'illustrateur qui dessine les personnages, séquence et découpe les actions, choisit la taille et la disposition des cases dans la page, impose son style graphique, choisit les éléments de décor, etc.

L'analyse du contenu d'une BD doit donc laisser toute sa place à ce travail de graphiation et questionner son articulation au travail de narration discursive (choix des dialogues et des commentaires de case). La tentation est grande de réduire cette articulation entre images et discours à une simple élucidation : le dialogue ou le commentaire serait là uniquement pour lever l'ambiguïté de l'image, par nature plus complexe à analyser car d'interprétation plus ouverte (Gaudreault, Jost, 1990). L'image est alors réduite à une simple illustration dont l'interprétation dépend de la lecture du texte écrit. Cette subordination de l'image au discours a été vivement dénoncée dans le champ de la bande dessinée par une certaine critique et par différents auteurs (Baetens, 1998, p. 47). L'autonomisation de l'image par rapport à l'écrit fait donc partie d'un discours normatif sur la bande dessinée valorisant la mise en retrait, voire la disparition du texte au profit de l'image.

D'un point de vue plus analytique, il convient de considérer le réductionnisme discursif qui cantonne l'image à un rôle illustratif et le rejet de tout discours comme les deux pôles opposés d'un continuum de relations possibles entre image et discours. Entre ces deux extrêmes se déploie un large spectre de relations possibles entre iconographie et texte. Par exemple, une image peut venir compléter un texte, et même à rebours de l'hypothèse réductionniste, éclairer un texte ambigu. Dans Quai d'Orsay, l'image vient parfois s'opposer au texte, le contraste entre les deux faisant naître une troisième signification. L'analyse présentée ici se veut attentive à la multiplicité des relations image/ texte qui se nouent dans les cases d'une bande dessinée.

À la lecture de ces premiers développements, il pourrait paraître opportun de désigner la vignette ou la case ${ }^{6}$, lieu de rencontre entre l'image et le texte, comme l'unité de base de l'analyse de contenu d'une BD. Ce serait courir le risque de ne

6. Le terme de case sera ici employé par convention : l'analyse fera apparaître que Blain joue sur les frontières de chaque dessin et n'utilise pas toujours à strictement parler des cases aux contours bien délimités. 
pas saisir cette case dans sa relation avec les vignettes passées ou à venir, partiellement disponibles au regard du lecteur (Peeters, 1993, p.15). La signification d'une case dépend de son positionnement dans une séquence où plusieurs cases se répondent entre elles. Ces relations ne se limitent pas toujours à un lien de cause à effet ou à une simple consécution (une même action découpée en $x$ vignettes). La plupart des dessinateurs recourent à des formes de «tressage» (Groensteen, 2007) beaucoup plus subtiles : "Participe du tressage tout ce qui instaure une relation remarquable (c'est-à-dire allant au-delà de la simple relation de causalité et de consécution) entre deux ou plusieurs vignettes. Le tressage définit une série [...] à l'intérieur de la trame séquentielle» (ibid., p.50).

La relation image/texte doit donc être analysée dans la continuité de trames ou «tresses » narratives. En effet, l'analyse de la manière dont les personnages du ministre et de son directeur de cabinet sont construits ne peut se mener au niveau de la case : c'est l'enchaînement des vignettes qui permet au lecteur de se faire une idée à leur sujet.

\section{Le tressage d'une opposition : le ministre et le chef de cabinet}

Les personnages du ministre (Taillard de Vorms) et du chef de cabinet (Maupas) constituent les deux pôles entre lesquels oscille le héros (Vlaminck) et plus largement le récit de Quai d'Orsay. Notre étude a naturellement porté sur l'ensemble de l'ouvrage; cependant, pour la commodité de la présentation, nous isolerons ici trois planches (voir en annexe) illustrant les trois types de tressage de ces deux personnages que l'analyse a permis d'isoler. Le lecteur trouvera systématiquement en note les références des autres pages qui s’inscrivent dans le même type de tressage.

Cette analyse fait apparaître une opposition forte dans la manière dont les personnages sont fictionnalisés. Cette opposition apparaît clairement dans le dernier type de tressage, basé non pas sur la succession mais sur l'opposition de séries de vignettes où les deux personnages apparaissent séparément sur une même planche (une forme de tressage croisé des personnages qui invite à la comparaison).

\section{La violence de l'expression discursive et gestuelle du ministre}

La planche 1 (annexe) isole une séquence du récit assez illustrative de la relation entre les trois personnages. Vlaminck soumet un projet de discours au ministre (première case), qui réagit négativement. Le novice est d'autant plus interloqué (voir la graphiation du personnage dans la troisième case) qu'il a l'impression d'avoir suivi les consignes que Taillard de Vorms lui avait données sous forme de mots clés : «responsabilité, unité, efficacité». Or le ministre 
évoque désormais un autre triptyque : « légitimité, lucidité, efficacité». Comme souvent dans le récit, le chef de cabinet se pose en médiateur entre le ministre et le novice, défendant la cause de ce dernier et endossant une partie de la paternité et de la responsabilité du discours (« tu vois, Alexandre, c'est un peu ce qu'on a fait»). Malgré cette intervention, le ministre se lance dans une critique féroce de la structuration du discours.

Du point de vue de la graphiation, les quatre dernières cases (ainsi que la première) offrent un excellent exemple de la façon dont le personnage du ministre est de manière récurrente mis en image dans cette bande dessinée?. Taillard de Vorms effectue des mouvements secs, hachés, martelés, signifiés par des dessins de nuages ou des coups de crayons figurant le vent déplacé par ses gestes brusques. Ceux-ci apparaissent d'autant plus rapides et violents que chaque case décompose un mouvement, un peu comme sur ces photographies qui gardent la trace d'un mouvement trop rapide pour la vitesse de prise de vue. Au lieu d'être découpé en plusieurs cases, le mouvement des mains est en quelque sorte condensé dans une seule vignette, générant cet effet d'accélération. En outre, ces mouvements de mains diffèrent d'une case à l'autre de cette trame, donnant l'impression d'un personnage agité, à la gestualité foisonnante. Le fond de case, laissé en blanc, semble dessiner un espace libre où se déploie l'ampleur démesurée des gestes. L'absence de délimitation de ces cases ${ }^{8}$ renforce cette impression.

Le rapport entre iconographie et texte est ici double. Une partie du rapport texte/image vient renforcer l'impression que les gestes sont saccadés. Le recours aux onomatopées («ta-ca-ta-ca-tac» ou «tchac, tchac, tchac»), le soulignement des mots ("légitimité, lucidité, efficacité»), la multiplication des phylactères dans la première case de la trame (traduction graphique d'une parole hachée) constituent autant de ponts entre graphiation et discours. Certains énoncés renforcent cette impression de violence gestuelle : « il faut marteler!», «ll faut enfoncer le clou», «Les gens, c'est des cons». Cependant la relation texte/image ne peut être ramenée ici à une simple congruence autour de l'idée de mouvements hachés et violents. En effet, cette graphiation est également mise en relation avec des phylactères qui contiennent des mots abstraits, sortis de tout contexte : « légitimité, lucidité, efficacité ». À la croisée de la graphiation du mouvement et de ces énoncés abstraits naît une signification nouvelle. Le ministre brasse du vent, à la fois littéralement (le vent représenté graphiquement) et métaphoriquement : il utilise en effet des mots creux, interchangeables (ne parlait-il pas auparavant de «responsabilité, unité, efficacité »?) et dénués de rapport avec une quelconque réalité concrète.

Pour autant, cela ne signifie pas que la parole du ministre ne puisse à

7. On retrouve le même type de tressage : p. 5, 11, 14, 15, 26, 29, 38, 39, 53, 78, 79.

8. Ces limites sont tracées à d'autres moments du récit. 
d'autres moments se révéler séductrice pour son entourage technocratique. Les idées qu'il brasse peuvent à l'occasion être regardées comme constitutives d'une «vision» des relations diplomatiques (p. 6-7 en particulier). Il faut noter ici que ce n'est pas la compétence technocratique du ministre, sa connaissance intime des dossiers, qui fonde cette admiration (Quai d'Orsay montre bien que le ministre ne s'embarrasse pas avec ces détails) 9 mais sa capacité à incarner, par le souffle de sa parole, une vision des intérêts diplomatiques de la France (voir le succès du discours du ministre en Allemagne, p.59). La "grandeur» du ministre s'inscrit ainsi dans le cadre de la «cité inspirée »10 (Boltanski, Thévenot, 1991) : un membre de son cabinet le décrit d'ailleurs comme un "génie» qui « a un temps d'avance ${ }^{11}$ sur tout le monde » (p.36). Ceci nous amène à donner une seconde interprétation, non exclusive de la première mais sur un mode mineur, du «vent» que génère en permanence Taillard de Vorms : ce mouvement d'air incarne également le "souffle» du personnage, sa capacité à amener dans son sillage des individus convaincus par son panache et sa façon originale de voir les relations internationales.

\section{L'économie de gestes et la discrétion du directeur de cabinet}

La planche 2 (annexe) nous présente un autre tressage ${ }^{12}$ qui intervient à un moment ultérieur du récit, quand le chef de cabinet se trouve confronté à une crise diplomatique majeure (un bateau militaire français, en flammes, menace un port du royaume imaginaire de Lousdem). Cette trame représente la manière dont le chef de cabinet gère cette crise. Il apparaît dans chaque case avec un visage fermé, signe que l'on peut interpréter en fonction du contexte narratif comme marquant à la fois sa préoccupation et son extrême concentration. Le fond blanc, ici, ne permet pas de souligner le mouvement mais de mettre en valeur l'immobilité du personnage ainsi que certains objets : un téléphone fixe, un téléphone portable, un ordinateur, un coffre-fort que l'on imagine rempli de dossiers diplomatiques, un de ces dossiers secrets probablement sortis de ce coffre, un téléphone sécurisé installé dans une mallette. Ces objets, nettement détachés sur le fond blanc, ne sont pas de simples éléments de décor : ils

9. Le père du ministre, qui est aussi une de ses sources d’inspiration, peut ainsi dire : « Les détails, il faut qu'il laisse ça aux technocrates» (p. 51).

10. Dans cette cité, c'est l'inspiration intérieure (celle du prophète, de l'artiste, de l'innovateur) qui définit la grandeur, laquelle n'est pas toujours comprise par l'opinion.

11. Son intuition des relations internationales peut amener le ministre à soutenir une position contre l'opinion dominante : c'est le cas par exemple dans le tome II de Quai d'Orsay où Taillard de Vorms soutient, seul contre tous ou presque, l'idée qu'il n'y a pas d'armes de destruction massive au Lousdem et que les Américains mentent quand ils affirment le contraire. On retrouve ici la figure, centrale dans la "cité inspirée», du "génie incompris» qui défend sa conviction intérieure contre l'opinion, conviction qui se révélera sans doute justifiée dans la suite du récit (l'Irak apparaissant ici clairement comme le modèle du Lousdem).

12. On retrouve des tressages similaires p. 40, 41, 42, 45 . 
sont les attributs qui nous permettent de qualifier l'action menée par le chef de cabinet. Ils nous permettent de comprendre que Maupas se livre à une négociation délicate, lourde de secrets d'État et qui nécessite des protections particulières (une conversation protégée).

Dans deux cases, le chef de cabinet se livre à deux actions en même temps : téléphoner avec deux combinés à la fois, téléphoner et écrire sur son ordinateur. La graphiation ne laisse aucun doute sur le fait qu'il s'agit d'actions simultanées et non pas, comme dans la planche 1 , de la trace de mouvements rapides successifs (absence de graphiation de vent). L'impression générale est non plus celle d'un homme agité mais celle d'un homme affairé, capable de faire deux choses à la fois quand la situation l'exige. L'impression d'affairement est renforcée par l'absence de cadre venant délimiter les vignettes, absence qui souligne l'enchaînement des actions ${ }^{13}$. Si, au fil de la trame, le chef de cabinet passe d'une action à l'autre, il garde le même air sérieux : il apparaît à la fois actif et calme face à cette situation extrême.

Dans cette trame, aucun texte : la graphiation est ici autonome. Tout se passe comme si les actes du chef de cabinet parlaient pour lui. Son action n'appelle ni commentaire ni explicitation des échanges. Alors que le ministre se paie de mots, le chef de cabinet agit. Alors que Taillard de Vorms est dans l'abstrait, Maupas est dans le concret. À un autre niveau de signification, le silence discursif reflète le secret, le non-dit, la non-publicité qui sont au cœur de la pratique diplomatique. Le secret des chancelleries contraste alors avec les discours flamboyants (ou qui se veulent tels) du ministre, déconnectés d'une réalité non explicitable (dans la mesure en particulier où elle mettrait en avant des motifs ou des moyens d'action perçus comme illégitimes). L'activité diplomatique se déploie donc pour partie dans des «arcanes » (Senellart, 2003) qui contrastent avec la visibilité représentationnelle du ministre.

\section{Violence et contrôle de soi : représentations conjointes du ministre et du chef de cabinet}

Cette opposition récurrente entre le ministre et le chef de cabinet se manifeste parfois sur la même planche, comme c'est le cas dans la planche $3^{14}$. Ce tressage complexe est composé de deux éléments. Jouant sur la composition de la page, Blain oppose une séquence centrale, qui découpe une action de haut en bas : le chef de cabinet raccroche, ouvre une porte puis s'avance dans un couloir du ministère. La séquence est encore une fois silencieuse, à l'exception d'une onomatopée dont le rôle est simplement de souligner un acte graphié (le «clac»

13. On voit ici que des dispositifs de graphiation semblables (fond blanc, absence de délimitation des cases) peuvent servir des buts dissemblables, voire opposés.

14. Le même dispositif de tressage se retrouve p.46, 54 et 55 . 
du téléphone que l'on raccroche). Le chef de cabinet paraît serein, sérieux : ses gestes ont une vocation instrumentale claire (raccrocher, ouvrir la porte). Dans les deux dernières cases, les mains, inutiles, restent dans les poches.

Les cases latérales découpent une autre action où le ministre joue un rôle central. Face à une secrétaire, il évoque sa méthode de travail : le «stabilossage ». Il explique qu'il utilise un stabilo pour souligner les parties intéressantes d'un livre et d'un discours et que par conséquent, le niveau de «stabilossage » (surlignage) est un indicateur fort de l'intérêt qu'il trouve à un écrit. Le discours du novice, peu surligné, est donc mauvais selon le ministre. Discursivement, cette séquence nous offre une clé de lecture importante pour le personnage. De manière évidente, le discours sur le stabilo définit le ministre non pas comme un auteur mais comme un simple (re)lecteur. II n'est l'auteur ni des idées qu'il exprime (il les puise dans les livres surlignés) ni de ses discours (qu'il fait écrire par d'autres, ici Vlaminck). De manière plus subtile, le surlignage est associé à une activité de surface, superficielle : «Un livre, pour savoir s'il est bien, j'ai à peine besoin de le lire. Je le stabilosse intuitivement. » Le ministre semble rester à la surface des choses, ne rien approfondir avec sérieux. Du point de vue de la graphiation, on retrouve des mouvements violents, soulignés à la fois par des nuages de vent et des onomatopées ("Vlaf», «Vrrlaaf», «Vrlouuf») qui soulignent le déplacement d'air. À l'articulation du discursif et du graphié on observe, comme dans la planche 1, un découpage des phylactères qui hache la parole du ministre, lui donnant un aspect nerveux et saccadé. De plus, la violence voire la vulgarité du discours ("ça m’énerve»; «ça veut dire qu'il est à chier»; "ça m'emmerde tout ça») vient articuler une violence symbolique à la violence physique des mouvements (jet d'un livre sur une table, bureau frappé du plat de la main).

In fine, la violence des gestes (qui montre à quel point le ministre entend imposer son point de vue à la secrétaire) souligne par contraste la vacuité du propos : Taillard de Vorms s'énerve à propos de stabilos usagés alors qu'il faut gérer une crise au Lousdem (crise diplomatique mise sur le même plan que des problèmes conjugaux ou parentaux). Encore une fois, le ministre fait beaucoup de bruit et de vent pour rien...

Le tressage de cette planche fonctionne sur l'opposition de ces deux séquences. L'économie de geste, et encore plus de parole, du chef de cabinet contraste avec la profusion de mouvements et de discours du ministre. Prise dans son ensemble, la trame souligne à la fois l'impulsivité et la superficialité de ce dernier face au contrôle de soi ${ }^{15}$ et au discernement du chef de cabinet.

15. Bien entendu, il arrive au chef de cabinet de paraître abasourdi (surtout quand Taillard de Vorms le surprend par ses réflexions déconnectées des réalités diplomatiques - par exemple p. 80 ou 87), surmené (il est alors dessiné avec des cernes ou avec une barbe naissante) voire un peu dépassé (par exemple p. 10, 12 ou 13). Cependant, même dans ces situations, il ne se départit jamais de son calme, reste poli et garde le contrôle de son corps (absence de grands gestes). 
Cette impression est renforcée par le jeu sur la limitation des cases : alors que celles qui découpent l'action du chef de cabinet sont clairement délimitées (suggérant une action «cadrée», "contrôlée»), les vignettes qui concernent le ministre n'ont pas de cadre et laissent libre cours à ses gestes impulsifs. Le récit ne fait que conforter cette interprétation : dès la page suivante le lecteur apprendra que la marche silencieuse de Maupas le conduit vers le bureau du ministre où il vient annoncer le dénouement heureux de la crise au Lousdem, qu'il a lui-même gérée. Alors que le ministre «stabilossait» des livres et le discours d'Arthur, le directeur de cabinet était en première ligne. Le chef de cabinet est du côté de l'efficacité gestionnaire, le ministre du côté de la mise en scène. L'homme de dossier et d'action est clairement opposé à l'homme des discours. II n'est d'ailleurs pas interdit de se demander si le nom «Maupas» ne doit pas se lire à l'envers : «pas de mots».

Cependant, Taillard de Vorms n'est pas toujours décrit dans Quai d'Orsay comme un personnage en retrait de l'action ou de la décision et qui laisserait son cabinet gérer les affaires diplomatiques. Ailleurs dans le récit, il apparaît particulièrement actif dans le traitement d'une autre crise, un conflit interethnique dans la «République démocratique de l'Oubanga». Mais son action paraît excessivement fougueuse : il décide, apparemment sur un coup de tête et sans tenir compte des risques qu'il encourt, de partir en avion sur place (p. 87), alors même que son chef de cabinet l'avertit du «chaos » qui règne dans la capitale de l'Oubanga. En outre, son intervention semble peu efficace ${ }^{16}$ : c'est Maupas qui in fine va régler la crise en appelant les chefs d'État de la région depuis son bureau parisien (p. 89). Autrement dit on retrouve, y compris dans le registre de l'action, l'opposition entre le visible et le spectaculaire (la télévision couvre le voyage de Taillard de Vorms en Oubanga), placés du côté du ministre, et le secret allié à l'efficacité, placé du côté de son chef de cabinet.

\section{L’Âge d'or diplomatique et la tentation de la communication}

Quai d'Orsay contribue à mettre en lumière les conditions de production de la parole ministérielle. En cela, cette œuvre a un véritable intérêt sociologique, remettant en cause certaines des évidences sur lesquelles nous fondons notre relation au discours politique. Cependant, l'analyse du tressage entre les personnages du ministre et du chef de cabinet nous permet de comprendre que Quai d'Orsay n'est pas une simple entreprise de dénaturalisation et de déconstruction du discours politique mais expose un point de vue normatif sur la communication politique en valorisant la discrétion, la prise de

16. D'une certaine façon, on peut dire que l'action du ministre «brasse de l'air» en ce qu'elle ne débouche pas sur grand-chose de concret. 


\begin{tabular}{|c|c|c|c|c|}
\hline Personnages & Graphiation & Texte & $\begin{array}{l}\text { Relation } \\
\text { images/ } \\
\text { discours }\end{array}$ & Signification \\
\hline \multirow[b]{2}{*}{ Le ministre } & \multirow{2}{*}{$\begin{array}{l}\text { Mouvements } \\
\text { violents } \\
\text { (corps, main) } \\
\text { Épaules hautes } \\
\text { (le ministre } \\
\text { "porte beau», } \\
\text { en impose) }\end{array}$} & $\begin{array}{l}\text { Violence } \\
\text { (vulgarité, } \\
\text { onomatopées) }\end{array}$ & $\begin{array}{l}\text { Redondance } \\
\text { (la violence } \\
\text { du discours } \\
\text { correspond } \\
\text { à la violence } \\
\text { des gestes) }\end{array}$ & \multirow{2}{*}{$\begin{array}{l}\text { Être impulsif, } \\
\text { fougueux, coupé } \\
\text { du traitement } \\
\text { concret des } \\
\text { dossiers. } \\
\text { Le ministre } \\
\text { brasse du vent }\end{array}$} \\
\hline & & $\begin{array}{l}\text { Superficialité } \\
\text { (stabilossage) } \\
\text { Distance } \\
\text { au «terrain » } \\
\text { (concepts } \\
\text { abstraits) }\end{array}$ & $\begin{array}{l}\text { Contraste entre } \\
\text { la vacuité/ } \\
\text { abstraction } \\
\text { du propos et } \\
\text { la violence } \\
\text { graphique } \\
\text { avec laquelle } \\
\text { ils sont assénés }\end{array}$ & \\
\hline \multirow[b]{2}{*}{$\begin{array}{l}\text { Le chef } \\
\text { de cabinet }\end{array}$} & $\begin{array}{l}\text { Économie } \\
\text { de geste } \\
\text { Présence } \\
\text { d'attributs } \\
\text { du «sérieux» et } \\
\text { de la profondeur }\end{array}$ & $\begin{array}{l}\text { Économie de la } \\
\text { parole qui peut } \\
\text { aller jusqu'à son } \\
\text { absence, contrôle } \\
\text { du discours (laco- } \\
\text { nique, poli) }\end{array}$ & $\begin{array}{l}\text { La graphiation } \\
\text { tend } \\
\text { à s'autonomiser } \\
\text { de l'écrit: } \\
\text { les gestes } \\
\text { parlent pour } \\
\text { le personnage }\end{array}$ & \multirow{2}{*}{$\begin{array}{l}\text { Être rationnel, } \\
\text { gestionnaire, } \\
\text { sous contrôle, } \\
\text { conscient } \\
\text { de ses } \\
\text { responsabilités, } \\
\text { homme } \\
\text { de dossier }\end{array}$} \\
\hline & $\begin{array}{l}\text { stabilo mais } \\
\text { des téléphones, } \\
\text { des ordinateurs, } \\
\text { des dossiers } \\
\text { secret défense) } \\
\text { Épaules basses } \\
\text { (il a tout le } \\
\text { poids de ses } \\
\text { responsabilités } \\
\text { sur ses épaules) }\end{array}$ & $\begin{array}{l}\text { Référence à des } \\
\text { faits concrets } \\
\text { (crise } \\
\text { diplomatique) }\end{array}$ & $\begin{array}{l}\text { Contraste entre la } \\
\text { crise évoquée dis- } \\
\text { cursivement et la } \\
\text { maîtrise du corps } \\
\text { et des gestes } \\
\text { (signe de calme) }\end{array}$ & \\
\hline
\end{tabular}

Tableau 1. Le tressage entre le personnage du ministre et celui du chef de cabinet 
distance par rapport au champ médiatique en tant qu'idéal de fonctionnement pour un cabinet ministériel.

À un premier niveau de lecture, Quai d'Orsay contribue au dévoilement des coulisses de la communication politique par l'élucidation du travail collectif que nécessite la production discursive d'un ministre. Cette bande dessinée montre que le ministre n'est pas à strictement parler l'auteur de ses discours. Pour autant, ArthurVlaminck ne peut pas être considéré non plus comme "l'écrivain » des prises de parole ministérielles ${ }^{17}$. Il occupe plutôt une position transitive «d'écrivant " (Ollivier-Yaniv, 2003, 2011) : il est celui qui articule des influences multiples et souvent divergentes en un discours cohérent. Il se fait «interprète» et «traducteur» de registres de langage différents (OllivierYaniv, 2003) : le registre technique des conseillers thématiques qu'il doit vulgariser (au risque d'être accusé de dénaturer leurs propos), le registre abstrait et abscons du ministre et de son entourage (des philosophes, des poètes, le propre père de Taillard de Vorms). Quai d'Orsay montre bien comment le discours ministériel se construit autour d'un cycle itératif, ce qui rejoint les observations de Caroline Ollivier-Yaniv :

D’abord la prise de parole ministérielle est rédigée par l'écrivant à partir de différentes sources telles les notes d'un ou plusieurs conseillers, ses propres lectures, ainsi qu'en fonction du public à qui le ministre va s'adresser. Se déroulent ensuite des séries d'aller-retour avec le directeur de cabinet et avec le ministre pour rectifications éventuelles. Selon l'importance du texte, les allers-retours peuvent se multiplier [...]. (Ibid., p. 93)

La parole ministérielle apparaît donc comme une œuvre collective issue d'un processus tant collaboratif que conflictuel entre des acteurs multiples. Quai d'Orsay participe donc à la fois à la dénonciation de «l'illusion du locuteur (politique) auteur de son discours» (Le Bart, 2010, p. 79) et à celle, symétrique, d'une prise de parole publique uniquement contrôlée par les «communicants » (Nollet, 2006).

Cependant, les auteurs de Quai d'Orsay ne se contentent pas de mettre en avant le caractère collectif de la production du discours politique : autour de l'opposition entre Maupas et Taillard de Vorms se joue une certaine disqualification de la communication politique, activité qui n'est pas ici réduite aux prises de parole publiques puisqu'elle inclut l'action spectaculaire (l'action qui se donne à voir mais qui ne produit pas nécessairement beaucoup d'effets concrets). Le ministre développe ainsi une approche cynique de la communication. Les critiques qu'il exprime par rapport aux discours rédigés par Vlaminck dans la planche 1 ne font pas référence à un quelconque message à

17. Il n'apparaît pas comme le véritable auteur du discours (de même que certains romanciers ont leurs nègres), isolé de toute influence, mais plutôt comme celui qui synthétise et met en intrigue les discours (plus ou moins contradictoires) des autres. 
faire passer mais à la façon dont le message doit être passé. Ce qui intéresse le ministre, c'est de communiquer de manière efficace (pour cela, «il faut marteler») face un public à l'attention et à l'intellect supposés limités («les gens, c'est des cons »). De la même façon, les consignes de Taillard de Vorms à l'attention de Vlaminck (« légitimité, lucidité, efficacité »; « responsabilité, unité, efficacité ») sonnent souvent comme des slogans ou comme des formules rhétoriques que l'on pourrait appliquer indifféremment à n'importe quel sujet. Est alors pointée l'obsession formelle du ministre qui paraît plus préoccupé par le style que par le contenu de son discours. Dans un autre registre, Quai d'Orsay souligne la volonté du ministre de porter un discours public «original» et «audacieux» (voir en particulier p.51-55), doté d'un souffle intellectuel et émotionnel : il ne veut pas d'une communication compassée, technique, prudente qu'il assimile, insulte suprême, à un "salmigondis de technocrates» (p.54). Il semble donc militer pour un certain affranchissement de la communication publique vis-à-vis des contraintes technocratiques.

Dans Quai d'Orsay, cette vision de la communication politique s'accompagne, en miroir, d'une valorisation du rôle silencieux du cabinet, et en particulier de celui de son chef. En effet, les tressages que nous avons étudiés mettent en regard le «brassage de vent » ministériel et l'efficacité, la connaissance du terrain, la compétence technique de Maupas. Le ministre est du côté des grands gestes, des grands mots, de l'action spectaculaire alors que le travail concret se fait loin des caméras et des micros. Il y a ici une association forte entre efficacité, compétence et discrétion comme si toute bonne diplomatie devait passer par le secret. Le directeur de cabinet, par opposition à Taillard de Vorms qui stigmatise le manque de fougue et la prudence du discours technocratique, est un homme de l'ombre qui valorise la discrétion jusque dans son comportement physique et fuit l'exposition et les médias. Ce rejet ou, plus exactement, ce désir de contrôle de la communication ${ }^{18}$ transparaît clairement dans une planche où Maupas tance vertement les membres de son cabinet quand une fuite est publiée dans les journaux:

Comme vous le savez, il y a eu une fuite dans la presse. Le pire c'est que certains d'entre vous ont probablement dû parler puisqu'ils sont cités. Ceux dont je parle se reconnaîtront. C'est très grave et surtout très triste. Je sais qu'aujourd'hui personne ne résiste au plaisir de voir son nom cité dans le journal. Je viens d'une famille de diplomates et je peux vous dire que dès qu'il y en avait un qui avait son nom dans le journal, tout le monde se foutait de sa gueule. C'est qu'il était nul. (p. 41)

Comme la fin de cette citation le révèle, Quai d'Orsay participe de la construction du mythe d'un Âge d'or technocratique révolu. Maupas est l'héritier, au

18. Le cabinet semble avoir en partie pour fonction de canaliser la fougue du ministre, de contrôler et recadrer ses pulsions de communication et de décision. 
sens figuré et au sens propre ${ }^{19}$, d'une longue tradition de discrétion qui se perd. Les membres plus jeunes de son cabinet (le dessin le fait clairement apparaître comme le plus âgé) sont, eux, tentés par le démon de la communication, de l'exposition, de la célébrité. De facto, l'idéalisation de la compétence technocratique dans le récit vise surtout Maupas. Le reste du cabinet est décrit comme un panier de crabes où chacun poursuit ses objectifs personnels au détriment des autres. Quand Arthur Vlaminck est trahi par une de ses collègues qui vient critiquer derrière son dos un de ses discours, le conseiller Amérique, entreprenant de déniaiser le novice, lui décrit, à travers une métaphore sexuelle, la vie de cabinet comme une jungle où s'échangent des coups : «Ici, c'est comme ça qu'on fait l'amour [...]. Tu verras, tu y prendras goût aussi. Quand tu fais un coup de pute à quelqu'un, c'est pas que tu lui veux du mal. C'est comme une caresse. S'il est bon, il va te faire un coup de pute après. C'est une relation amoureuse.» (p. 20)

Maupas, tel un témoignage du passé glorieux de la diplomatie française, semble bien au-dessus de cette "sexualité de cabinet». Il traite les dossiers avec sang-froid et non avec sensualité, il ne cherche ni la publicité ni à faire des coups bas à Arthur Vlaminck qu'il prend sous son aile. Il est en quelque sorte l'incarnation (jusqu'à la caricature?) du mythe du technocrate : homme compétent, discret et dévoué à l'intérêt général.

Il est permis de se demander dans quelle mesure Quai d'Orsay ne reflète pas l'adhésion de son scénariste à ce mythe technocratique incarné par Maupas. Comme toute œuvre fictionnelle, Quai d'Orsay en dit sans doute autant sur l'objet décrit que sur celui qui l'a (co)produite. Ex-membre de cabinet, le scénariste expose un point de vue socialement situé sur le fonctionnement politique. Sont ici fortement distingués, conformément à la doxa de cabinet, un champ technocratique articulé autour de compétences techniques (en l'occurrence diplomatiques) qui se déploient dans l'idéal en toute discrétion, et le champ de la communication incarné par «l'écrivant» Arthur Vlaminck et le ministre, tourné vers la publicisation et la mise en récit de l'action. Cette distinction, qui tend à sous-évaluer la contribution indirecte des conseillers technique à l'élaboration des prises de parole publique, alimente ici un jugement de valeur: l'âge d'or perdu, c'est précisément celui où l'autonomie du champ diplomatique, confiné dans les chancelleries, n'était pas menacée par les impératifs de la communication et ses dangereux attraits ("le plaisir de voir son nom cité dans les journaux»). Quai d'Orsay constitue donc pour partie le reflet d'une vision nostalgique (et probablement mythique) de la division du travail politique où les technocrates savaient rester à l'écart de la tentation de la communication publique.

19. Il est fils de diplomate. 


\section{Références}

Blain Christophe, Lanzac Abel, 2010, Quai d'Orsay. Chroniques diplomatiques, t. I, Paris, Dargaud.

- 2011, Quai d'Orsay. Chroniques diplomatiques, t. II, Paris, Dargaud.

BoltANSKI Luc, THÉVENOt Laurent, 1991, De la justification. Les économies de la grandeur, Paris, Gallimard.

BAetens Jan, 1998, Formes et politique de la bande dessinée, Louvain, Peeters, Vrin.

Bon hомme Marc, 2010, "La caricature politique», Mots. Les langages du politique, n०94, p. 39-45.

Cohen Philippe, Malka Richard, 2007, La face karchée de Sarkozy, Paris, Vents d'Ouest.

GAud REAUlt André, Jost François, 1990, Le récit cinématographique, Paris, Nathan.

Groensteen Thierry, 2007, La bande dessinée, mode d'emploi, Liège, Les impressions nouvelles.

HeItz Bruno, 2010, J'ai pas tué de Gaulle mais ça a bien failli, Paris, Gallimard.

LE BART Christian, 2010, "Parler en politique », Mots. Les langages du politique, n 94 , p. 77-84.

McLuhan Marshall, 1977, Pour comprendre les médias. Les prolongements technologiques de l'homme, Paris, Le Seuil.

MARION Philippe, 1993, Traces en cases. Travail graphique, figuration narrative et participation du lecteur, Louvain-la-Neuve, Academia.

NolLet Jérémie, 2006, «Les communicateurs de ministère. Entre champs bureaucratiques et journalistiques", Journalisme et dépendances, I. Chupin, J. Nollet éd., Paris, L'Harmattan, p. 161-185.

Ollivier-Yaniv Caroline, 2003, "La fabrique du discours politique. Les “écrivants" des prises de parole ministérielles», Argumentation et discours politique. Antiquité grecque et latine, Révolution française, Monde contemporain, S. Bonnafous et al. éd., Rennes, Presses universitaires de Rennes, p. 89-98.

- 2011, «Petites phrases et éléments de langage. Des catégories en tension», Communication et langages, $\mathrm{n}^{0} 168, \mathrm{p}$.57-68.

Peeters Benoît, 1993, La bande dessinée, Paris, Flammarion.

SenelLART Michel, 2003, "Secret et publicité dans l'art gouvernemental des XVIIe et XVIII siècles », Quaderni, vol. LII, nº 1, p. 43-54. 

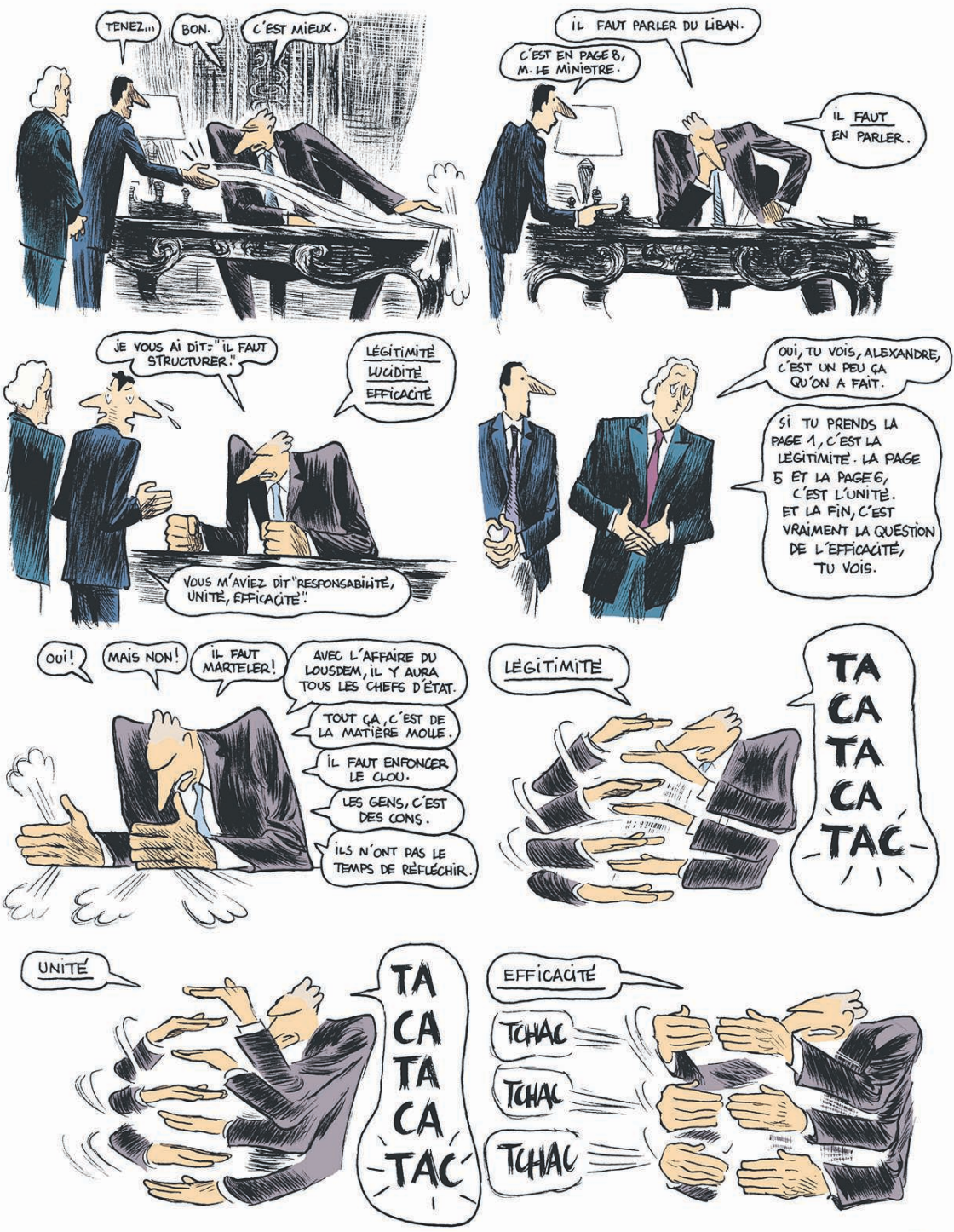

15

Planche 1. Le ministre brassant du vent (p. 15)

(C) Blain - Lanzac / Dargaud 2012 

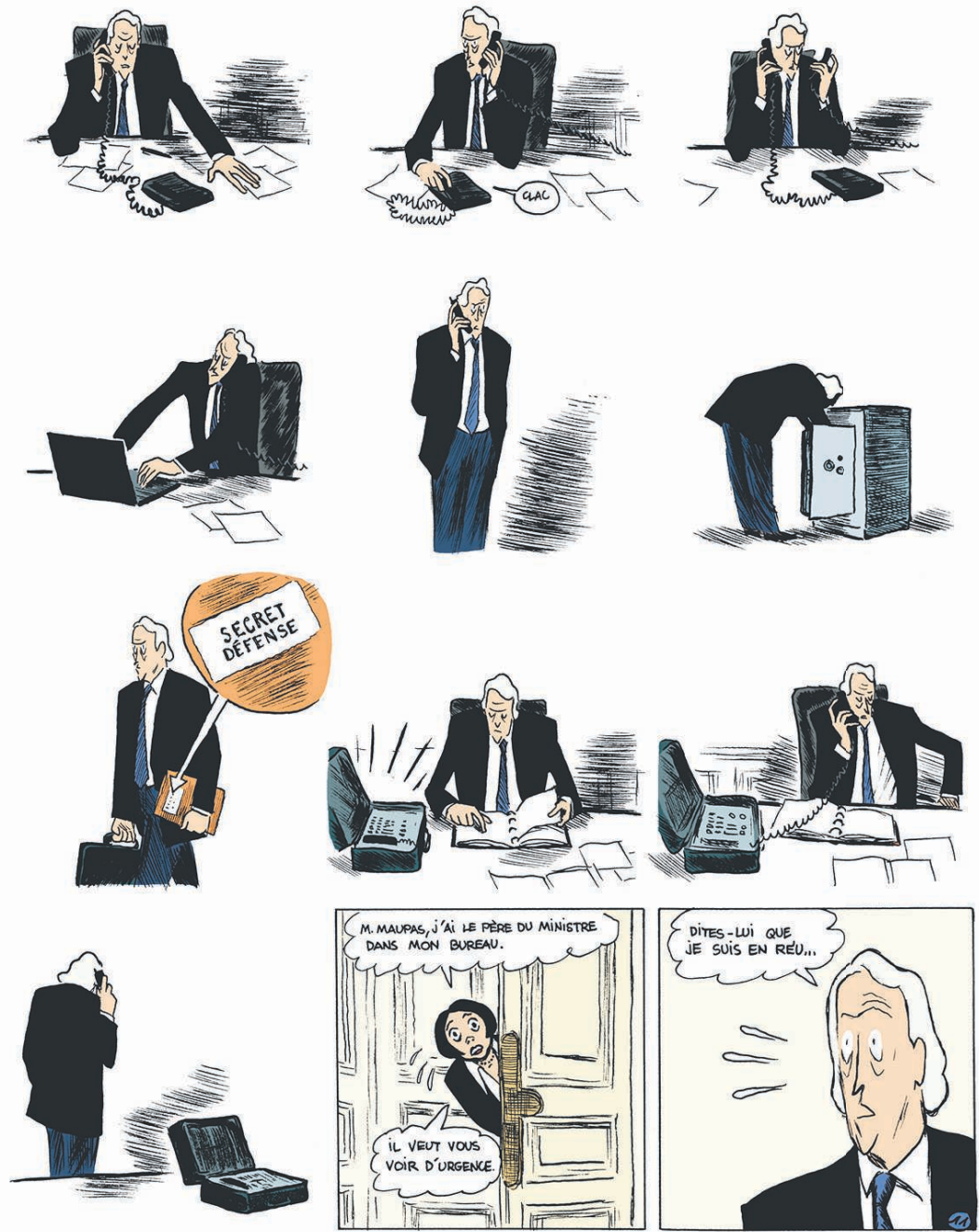

50

Planche 2. Le chef de cabinet en action (p. 50)

(C) Blain - Lanzac / Dargaud 2012 

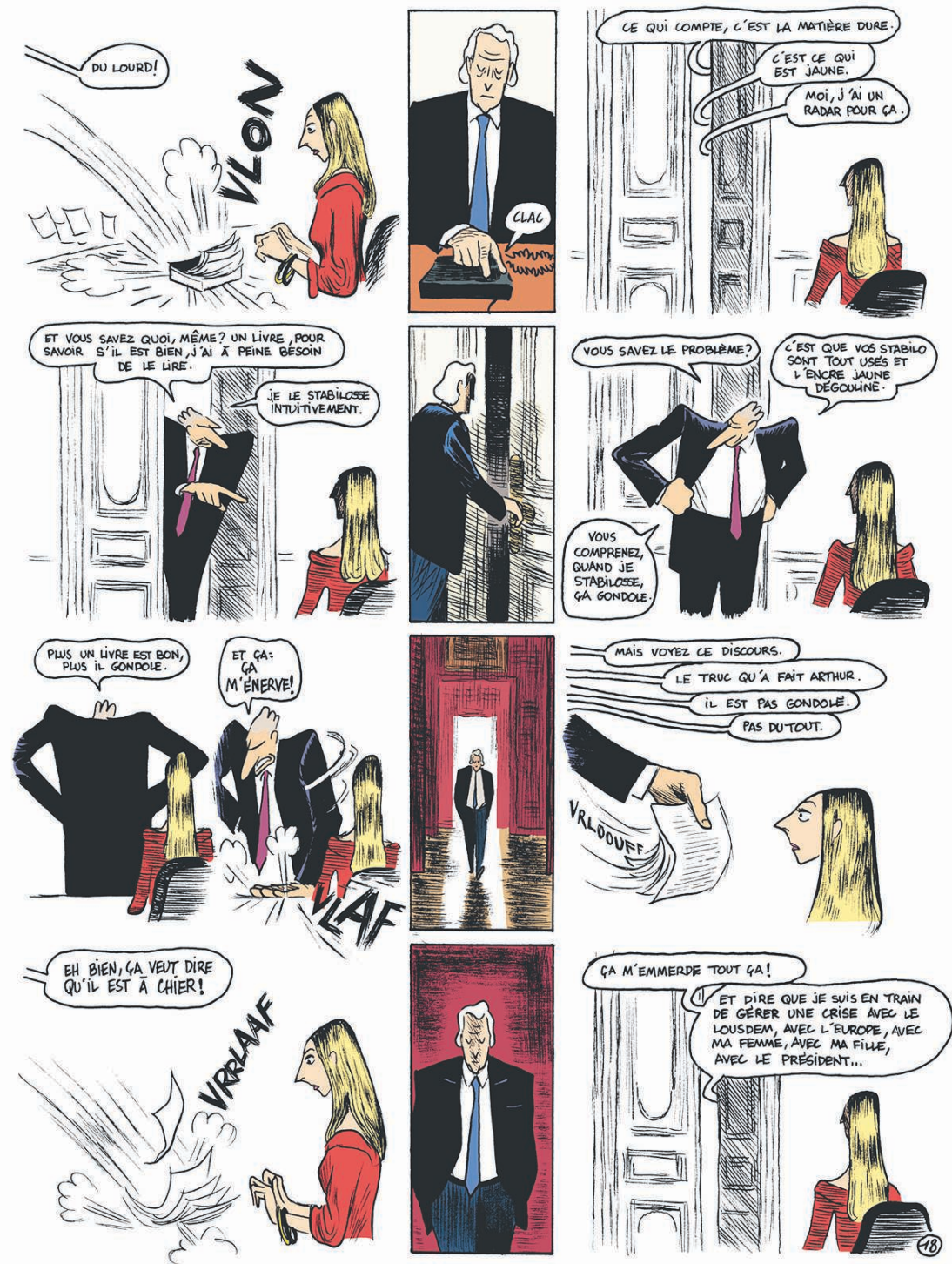

57

Planche 3. La virulence du ministre face au silence du chef de cabinet (p. 57) (C) Blain - Lanzac / Dargaud 2012 